\title{
COMPACT SPACE-LIKE HYPERSURFACES IN DE SITTER SPACE
}

\author{
JINCHI LV
}

Received 16 March 2005 and in revised form 7 June 2005

We present some integral formulas for compact space-like hypersurfaces in de Sitter space and some equivalent characterizations for totally umbilical compact space-like hypersurfaces in de Sitter space in terms of mean curvature and higher-order mean curvatures.

\section{Introduction}

It is well known that the semi-Riemannian (pseudo-Riemannian) manifolds $(M, g)$ of Lorentzian signature play a special role in geometry and physics, and that they are models of space time of general relativity. Let $M_{p}^{n+1}(c)$ be an $(n+1)$-dimensional complete connected semi-Riemannian manifold with constant sectional curvature $c$ and index $p$ (see [13, page 227]). It is called an indefinite space form of indexp and simply a space form when $p=0$. According to $c>0, c=0$, and $c<0, M_{1}^{n+1}(c)$ is called de Sitter space, Minkowski space, and anti-de Sitter space, and is denoted by $S_{1}^{n+1}(c), \mathbb{R}_{1}^{n+1}$, and $H_{1}^{n+1}(c)$, respectively. In spite of the fact that the geometry of de Sitter space is the simplest model of space time of general relativity, this geometry was not studied thoroughly. Let $\phi: M^{n} \rightarrow S_{1}^{n+1}(c)$ be a smooth immersion of an $n$-dimensional connected manifold into $S_{1}^{n+1}(c)$. If the semiRiemannian metric of $S_{1}^{n+1}(c)$ induces a Riemannian metric on $M^{n}$ via $\phi, M^{n}$ is called a space-like hypersurface in de Sitter space.

The study of space-like hypersurfaces in de Sitter space $S_{1}^{n+1}(c)$ has been of increasing interest in the last years, because of their nice Bernstein-type properties. Since Goddard [7] conjectured in 1977 that complete space-like hyperspaces in $S_{1}^{n+1}(c)$ with constant mean curvature $H$ must be totally umbilical, which turned out to be false in this original statement, an important number of authors have considered the problem of characterizing the totally umbilical space-like hypersurfaces in de Sitter space in terms of some appropriate geometric assumptions. Actually, Akutagawa [1] proved that Goddard's conjecture is true when $H^{2} \leq c$ if $n=2$, and $H^{2}<\left(4(n-1) / n^{2}\right) c$ if $n \geq 3$. On the other hand, Montiel [11] proved that Goddard's conjecture is also true under the additional hypothesis of the compactness of the hypersurfaces. We also refer to [14] for an alternative proof of both facts given by Ramanathan in the 2-dimensional case. More recently, Cheng and Ishikawa [5] have shown that compact space-like hyperspaces in $S_{1}^{n+1}(c)$ with constant 
scalar curvature $S<n(n-1) c$ must be totally umbilical. Aledo el al. [3] have recently found some other characterizations of the totally umbilical compact space-like hypersurfaces in de Sitter space with constant higher-order mean curvatures, under appropriate hypothesis.

In this paper, we will study various equivalent characterizations of totally umbilical compact space-like hypersurfaces in de Sitter space in terms of mean curvature and higher-order mean curvatures. The whole paper is organized as follows. Section 2 gives some preliminaries, Section 3 gives some inequalities on the normalized symmetric functions, and Section 4 reviews some selfadjoint second-order differential operator. The main results of this paper are contained in Section 5, which gives us a more specific and complete picture of totally umbilical compact space-like hypersurfaces in de Sitter space. For simplicity, we omit the volume form $d V$ in all integrals.

\section{Preliminaries}

We consider Minkowski space $\mathbb{R}_{1}^{n+2}$ as the real vector space $\mathbb{R}^{n+2}$ endowed with the Lorentzian metric $\langle\cdot, \cdot\rangle$ given by

$$
\langle x, y\rangle=-x_{0} y_{0}+\sum_{i=1}^{n+1} x_{i} y_{i}
$$

for $x, y \in \mathbb{R}^{n+2}$. Then de Sitter space $S_{1}^{n+1}(c)$ can be defined as the following hyperquadric of $\mathbb{R}_{1}^{n+2}$ :

$$
S_{1}^{n+1}(c)=\left\{\left.x \in \mathbb{R}_{1}^{n+2}|| x\right|^{2}=\frac{1}{c}\right\}
$$

The induced metric from $\langle\cdot, \cdot\rangle$ makes $S_{1}^{n+1}(c)$ into a Lorentzian manifold with constant sectional curvature $c$. Moreover, if $x \in S_{1}^{n+1}(c)$, we can put

$$
T_{x} S_{1}^{n+1}(c)=\left\{v \in \mathbb{R}^{n+2} \mid\langle v, x\rangle=0\right\} .
$$

We denote by $\nabla^{L}$ and $\bar{\nabla}$ the metric connections of $\mathbb{R}_{1}^{n+2}$ and $S_{1}^{n+1}(c)$, respectively. Then, we have

$$
\nabla_{v}^{L} w-\bar{\nabla}_{v} w=-c\langle v, w\rangle x
$$

for all $v, w \in T_{x} S_{1}^{n+1}(c)$. Let

$$
\phi: M^{n} \longrightarrow S_{1}^{n+1}(c)
$$

be a space-like hypersurface in $S_{1}^{n+1}(c)$ defined above. First, we want to know whether a compact one is orientable. The following proposition gives us the affirmative answer (see [11] or [2] for a proof).

Proposition 2.1. Let $\phi: M^{n} \rightarrow S_{1}^{n+1}(c)$ be a space-like hypersurface in $S_{1}^{n+1}(c), n \geq 2$. If $M^{n}$ is compact, then $M^{n}$ is diffeomorphic to $S^{n}$. In particular, compact totally umbilical space-like hypersurfaces in $S_{1}^{n+1}(c), n \geq 2$, are round $n$-spheres. 
Throughout the following, we will exclusively deal with compact space-like hypersurfaces in $S_{1}^{n+1}(c), n \geq 2$. The above proposition ensures that $M^{n}$ is orientable. Let $N$ be a time-like unit normal vector field for the immersion $\phi$. The field $N$ can be viewed as the Gauss map of $M^{n}$ into hyperbolic space:

$$
N: M^{n} \longrightarrow H^{n+1}
$$

where $H^{n+1}=\left\{\left.x \in \mathbb{R}^{n+2}|| x\right|^{2}=-1, x_{0} \geq 1\right\}$. We will say that $M^{n}$ is oriented by $N$. A well-known result is that the Gauss map $N$ is harmonic if and only if the mean curvature $H$ is constant. For a proof, one can refer to [4].

Let $\nabla$ be the Levi-Civita connection associated to the Riemannian metric on $M^{n}$ induced from $\langle\cdot, \cdot\rangle$. Then, we have

$$
\begin{aligned}
h(v, w) & =\bar{\nabla}_{v} w-\nabla_{v} w=-\langle\mathscr{A} v, w\rangle N \\
\mathscr{A} v & =-\bar{\nabla}_{v} N=-\nabla_{v}^{L} N
\end{aligned}
$$

where $\mathscr{A}$ stands for the shape operator of the immersion $\phi$ with respect to $N$ and $v, w$ are vector fields tangent to $M^{n}$. The operator $L=-\mathscr{A}$ is the Weingarten endomorphism. The eigenvalues of the operator $L$ are called the principal curvatures and will be denoted by $\lambda_{1}, \ldots, \lambda_{n}$. The Codazzi equation is expressed by

$$
\left(\nabla_{v} \mathscr{A}\right) w=\left(\nabla_{w} \mathscr{A}\right) v
$$

For a suitably chosen local field of orthonormal frames $e_{1}, \ldots, e_{n}$ on $M^{n}$, we have

$$
\mathscr{A} e_{i}=-\lambda_{i} e_{i}
$$

The $k$ th mean curvature of the space-like hypersurface $M^{n}$ is defined by

$$
H_{k}=\frac{1}{\left(\begin{array}{l}
n \\
k
\end{array}\right)} \sum_{i_{1}<\cdots<i_{k}} \lambda_{i_{1}} \cdots \lambda_{i_{k}}
$$

Note that when $k=1, H_{1}$ is the mean curvature $H$, and when $k=n, H_{n}$ is the GaussKronecker curvature. We can easily see that the scalar curvature

$$
S=n(n-1) c-\left(\sum_{i} \lambda_{i}\right)^{2}+\sum_{i} \lambda_{i}^{2}=n(n-1)\left(c-H_{2}\right)
$$

and the characteristic polynomial of $\mathscr{A}$ can be written in terms of the $H_{k}$ 's as

$$
\operatorname{det}(t I-\mathscr{A})=\sum_{k=0}^{n}\left(\begin{array}{l}
n \\
k
\end{array}\right) H_{k} t^{n-k}
$$

where $H_{0}=1$. 
Minkowski formulas provide us with a convenient tool in the study of hypersurfaces. One can refer to [12] for the well-known version for space forms. Many interesting results have been got in the study of hypersurfaces by means of Minkowski formulas, for example, $[9,10,12,16,17]$, and so forth. The proof in [12] followed the idea in [15]. Similar to it, one can easily give the proof of Minkowski formulas for compact space-like hypersurfaces in de Sitter space (see [3]). The following proposition is Minkowski formulas for compact space-like hypersurfaces in de Sitter space.

Proposition 2.2. Let $\phi: M^{n} \rightarrow S_{1}^{n+1}(c)$ be a compact space-like hypersurface in $S_{1}^{n+1}(c), n \geq$ 2 , then

$$
\int_{M^{n}} c H_{k}\langle\phi, a\rangle-H_{k+1}\langle N, a\rangle=0, \quad k=0,1, \ldots, n-1,
$$

for any $a \in \mathbb{R}^{n+2}$.

\section{Inequalities on the normalized symmetric functions}

Let $x_{1}, \ldots, x_{n} \in \mathbb{R}$. The elementary symmetric functions of $n$ variables $x_{1}, \ldots, x_{n}$ are defined by

$$
\sigma_{k}=\sum_{1 \leq i_{1}<\cdots<i_{k} \leq n} x_{i_{1}} \cdots x_{i_{k}}, \quad k=0,1, \ldots, n,
$$

where $\sigma_{0}=1$. For our purpose, it is useful to consider the normalized symmetric functions by dividing each $\sigma_{k}$ by the number of its summands. We denote the normalized symmetric function by

$$
E_{k}=\frac{1}{\left(\begin{array}{l}
n \\
k
\end{array}\right)} \sigma_{k}, \quad k=0,1, \ldots, n,
$$

where $E_{0}=1$. Since

$$
\left(x-x_{1}\right) \cdots\left(x-x_{n}\right)=\sum_{i=0}^{n}(-1)^{i} \sigma_{i} x^{n-i}=\sum_{i=0}^{n}(-1)^{i}\left(\begin{array}{l}
n \\
i
\end{array}\right) E_{i} x^{n-i},
$$

we see that at least $r$ of $x_{i}^{\prime}$ 's are zero if and only if $E_{n-r+1}=\cdots=E_{n}=0$.

Proposition 3.1. All $x_{i} \geq 0$ if and only if all $E_{i} \geq 0$, and all $x_{i}>0$ if and only if all $E_{i}>0$.

Proof. We prove it by induction on $n$. For $n=1$, the proposition holds clearly. Now assume that $n>1$ and the proposition holds for $n-1$. Let $P(x)=\left(x-x_{1}\right) \cdots\left(x-x_{n}\right)$ and $Q(x)=(1 / n) P^{\prime}(x)=\left(x-y_{1}\right) \cdots\left(x-y_{n}\right)$. By Rolle's theorem, $y_{1}, \ldots, y_{n-1}$ are all real and $x_{1} \leq y_{1} \leq x_{2} \leq \cdots \leq x_{n-1} \leq y_{n-1} \leq x_{n}$. Clearly, the inductive assumption applies to $y_{1}$, $\ldots, y_{n-1}$. Thus, it follows easily that the proposition holds for $n$.

There are some well-known inequalities on the normalized symmetric functions, for example, Newton-Maclaurin inequalities. One can refer to [8] for the case of $n$ positive numbers. For the sake of completeness, we include here a proof of Newton's inequalities for the general case. 
Proposition 3.2.

$$
E_{k}^{2} \geq E_{k-1} E_{k+1}, \quad k=1, \ldots, n-1,
$$

and each equality holds if and only if $x_{1}=\cdots=x_{n}$, or $E_{k}=0=E_{k-1} E_{k+1}$.

Proof. We prove it by induction on $n$. For $n=2$, the inequality holds clearly and the equality holds if and only if $x_{1}=x_{2}$ since $E_{1}=0=E_{0} E_{2}=E_{2}$ implies that $x_{1}=x_{2}=0$. Now assume that $n>2$ and the proposition holds for $n-1$. Let $P(x)=\left(x-x_{1}\right) \cdots(x-$ $\left.x_{n}\right)$ and $Q(x)=(1 / n) P^{\prime}(x)$. Then

$$
\begin{gathered}
P(x)=\sum_{i=0}^{n}(-1)^{i} \sigma_{i} x^{n-i}=\sum_{i=0}^{n}(-1)^{i}\left(\begin{array}{c}
n \\
i
\end{array}\right) E_{i} x^{n-i} \\
Q(x)=\frac{1}{n} P^{\prime}(x)=\sum_{i=0}^{n-1}(-1)^{i} \frac{n-i}{n}\left(\begin{array}{c}
n \\
i
\end{array}\right) E_{i} x^{n-i-1}=\sum_{i=0}^{n-1}(-1)^{i}\left(\begin{array}{c}
n-1 \\
i
\end{array}\right) E_{i} x^{n-1-i} .
\end{gathered}
$$

On the other hand,

$$
Q(x)=\left(x-y_{1}\right) \cdots\left(x-y_{n-1}\right)=\sum_{i=0}^{n-1}(-1)^{i}\left(\begin{array}{c}
n-1 \\
i
\end{array}\right) E_{i}\left(y_{1}, \ldots, y_{n-1}\right) x^{n-1-i},
$$

where $y_{1}, \ldots, y_{n-1}$ are $n-1$ roots of the polynomial $Q(x)$. Comparing the coefficients of the powers of $x$ in the above two expressions for $Q(x)$ gives us

$$
E_{i}\left(y_{1}, \ldots, y_{n-1}\right)=E_{i}\left(x_{1}, \ldots, x_{n}\right), \quad i=0, \ldots, n-1 .
$$

By Rolle's theorem $y_{1}, \ldots, y_{n-1}$ are all real. Clearly, $y_{1}=\cdots=y_{n-1}$ if and only if $x_{1}=$ $\cdots=x_{n}$. Thus the inductive assumption applies to $E_{i}\left(y_{1}, \ldots, y_{n-1}\right), i=0, \ldots, n-1$, and the proposition holds for $k=1, \ldots, n-2$ by (3.7).

It remains to prove for $k=n-1$, that is,

$$
E_{n-1}^{2}\left(x_{1}, \ldots, x_{n}\right) \geq E_{n-2}\left(x_{1}, \ldots, x_{n}\right) E_{n}\left(x_{1}, \ldots, x_{n}\right),
$$

with equality if and only if $x_{1}=\cdots=x_{n}$, or $E_{n-1}=0=E_{n-2} E_{n}$.

Case 1. If some $x_{i}=0$, then $E_{n}\left(x_{1}, \ldots, x_{n}\right)=x_{1} \cdots x_{n}=0$. Clearly, (3.8) holds with equality if and only if $E_{n-1}=(1 / n) \prod_{j \neq i} x_{j}=0$, and thus if and only if some $x_{j}=0, j \neq i$.

Case 2. If all $x_{i} \neq 0$, let $x_{i}^{\prime}=1 / x_{i}$. Then, we have

$$
\frac{E_{i}\left(x_{1}, \ldots, x_{n}\right)}{E_{n}\left(x_{1}, \ldots, x_{n}\right)}=E_{n-i}\left(x_{1}^{\prime}, \ldots, x_{n}^{\prime}\right) .
$$

Since $E_{n}\left(x_{1}, \ldots, x_{n}\right)=x_{1} \cdots x_{n} \neq 0$, we see that $(3.8)$ is equivalent to

$$
E_{1}^{2}\left(x_{1}^{\prime}, \ldots, x_{n}^{\prime}\right) \geq E_{2}\left(x_{1}^{\prime}, \ldots, x_{n}^{\prime}\right)
$$

which is true since $n>2$. 
This completes the proof.

Remark 3.3. For our future purpose, we concern most when each of the above equalities holds if and only if $x_{1}=\cdots=x_{n}$, that is, to find some restrictions on $x_{i}$ 's to exclude the possibility of $E_{k}=0=E_{k-1} E_{k+1}$ and $x_{i}$ 's are not all zero. We only know that $E_{1}^{2}=E_{2}$ holds if and only if $x_{1}=\cdots=x_{n}$ since $E_{1}=0=E_{0} E_{2}=E_{2}$ implies that $x_{1}=\cdots=x_{n}=0$, while we cannot expect it for $k \geq 2$ even if all $x_{i} \geq 0$, for example, when only one of $x_{i}$ 's is positive. In particular, when all $x_{i}$ 's have the same sign, that is, nonnegative or nonpositive simultaneously, and at least $k$ of $x_{i}$ 's are nonzero (equivalently, $E_{1} \cdots E_{k} \neq 0$ ) or $E_{1}=\cdots=E_{n}=0$, we have that $E_{k}^{2}=E_{k-1} E_{k+1}$ holds if and only if $x_{1}=\cdots=x_{n}$.

Newton's inequalities have a very important consequence, Maclaurin's inequalities, by investigating that

$$
E_{1}^{2} E_{2}^{4} \cdots E_{k}^{2 k} \geq\left(E_{0} E_{2}\right)\left(E_{1} E_{3}\right)^{2} \cdots\left(E_{k-1} E_{k+1}\right)^{k},
$$

where all $x_{i} \geq 0$. When all $x_{i}>0$ and $2 \leq k \leq n-1$, we have

$$
E_{k}^{1 / k} \geq E_{k+1}^{1 /(k+1)}
$$

with equality if and only if $x_{1}=\cdots=x_{n}$. If some of $x_{i}^{\prime}$ 's are zero and the rest of them are positive, then for $2 \leq k \leq n-1$, we still have

$$
E_{k}^{1 / k} \geq E_{k+1}^{1 /(k+1)}
$$

with equality if and only if $x_{1}=\cdots=x_{n}$, or at least $n-k+1$ of $x_{i}$ 's are zero.

Corollary 3.4. If all $x_{i} \geq 0,1 \leq k \leq n-1$, then

$$
E_{k}^{1 / k} \geq E_{k+1}^{1 /(k+1)}
$$

with equality if and only if $x_{1}=\cdots=x_{n}$, or $E_{n-k+1}=\cdots=E_{n}=0$.

Corollary 3.5. If $E_{1}>0, \ldots, E_{k}>0$ and $2 \leq k \leq n$, then

$$
E_{1} \geq E_{2}^{1 / 2} \geq \cdots \geq E_{k}^{1 / k}
$$

with each equality if and only if $x_{1}=\cdots=x_{n}$.

Now we can give a result on the positiveness of mean curvature and higher-order mean curvatures of the compact space-like hypersurfaces in de Sitter space.

Theorem 3.6. Let $\phi: M^{n} \rightarrow S_{1}^{n+1}(c), n \geq 2$, be a compact space-like hypersurface in de Sitter space with $H_{k}>0$ and $2 \leq k \leq n$. If there exists a point of $M^{n}$, where $H_{1}, \ldots, H_{k-1}$ are positive, then $H_{1}, \ldots, H_{k-1}$ are positive everywhere on $M^{n}$, that is, $H_{1}>0, \ldots, H_{k-1}>0$. 
Proof. We prove it by an open-closed argument. Let

$$
U=\left\{x \in M^{n} \mid H_{1}(x)>0, \ldots, H_{k-1}(x)>0\right\} .
$$

Clearly $U$ is open, and it is nonempty by the assumption. To prove that $U=M^{n}$, we only need to prove that $U$ is also closed by the connectedness of $M^{n}$. Since $H_{k}>0$ and $M^{n}$ is compact, we have

$$
a=\min _{x \in M^{n}} H_{k}(x)>0 .
$$

For any $x \in U$, we have

$$
H_{1}(x) \geq H_{2}(x)^{1 / 2} \geq \cdots \geq H_{k-1}(x)^{1 /(k-1)} \geq H_{k}(x)^{1 / k} \geq a^{1 / k}>0,
$$

by Corollary 3.5. Thus $U$ is closed. This completes the proof.

Finally, we give another two sets of important inequalities by investigating that

$$
\begin{aligned}
& E_{k}^{2} E_{k+1}^{2} \cdots E_{l}^{2} \geq\left(E_{k-1} E_{k+1}\right)\left(E_{k} E_{k+2}\right) \cdots\left(E_{l-1} E_{l+1}\right), \\
& E_{k}^{1 / k} \cdots E_{l-1}^{1 /(l-1)} E_{l}^{(l+1) / l} \geq E_{k+1}^{1 /(k+1)} \cdots E_{l}^{1 / l} E_{l+1},
\end{aligned}
$$

where all $x_{i} \geq 0$ and $1 \leq k<l \leq n-1$. Using the argument above leading to Corollary 3.4, we can get the following important inequalities.

Theorem 3.7. If all $x_{i} \geq 0$ and $1 \leq k<l \leq n-1$, then

$$
E_{k} E_{l} \geq E_{k-1} E_{l+1}
$$

with equality if and only if $x_{1}=\cdots=x_{n}$, or $E_{n-l+1}=\cdots=E_{n}=0$.

Theorem 3.8. If $E_{k-1}>0, \ldots, E_{l+1}>0$ and $1 \leq k<l \leq n-1$, then

$$
E_{k} E_{l} \geq E_{k-1} E_{l+1}
$$

with equality if and only if $x_{1}=\cdots=x_{n}$.

Theorem 3.9. If all $x_{i} \geq 0$ and $1 \leq k<l \leq n-1$, then

$$
E_{k}^{1 / k} E_{l} \geq E_{l+1}
$$

with equality if and only if $x_{1}=\cdots=x_{n}$, or $E_{n-l+1}=\cdots=E_{n}=0$.

Theorem 3.10. If $E_{1}>0, \ldots, E_{l+1}>0$ and $1 \leq k<l \leq n-1$, then

$$
E_{k}^{1 / k} E_{l} \geq E_{l+1}
$$

with equality if and only if $x_{1}=\cdots=x_{n}$. 


\section{Some selfadjoint second-order differential operators}

First, we introduce two known selfadjoint second-order differential operators, the Laplace operator $\triangle$ and the Cheng-Yau operator $\square$. For any $C^{2}$-function $f$ defined on $M^{n}$, we consider the symmetric bilinear form

$$
\left(\nabla^{2} f\right)(w, v)=v(w f)-\left(\nabla_{v} w\right) f
$$

The Laplace operator $\triangle$ acting on any $C^{2}$-function $f$ defined on $M^{n}$ is given by

$$
\triangle f=\sum_{i}\left(\nabla^{2} f\right)\left(e_{i}, e_{i}\right)
$$

Since $M^{n}$ is compact and oriented, the Laplace operator $\triangle$ is selfadjoint relative to the $L^{2}$-inner product of $M^{n}$, that is,

$$
\int_{M^{n}} f(\triangle g)=\int_{M^{n}}(\triangle f) g
$$

Following Cheng and Yau [6], we introduce an operator $\square$ acting on any $C^{2}$-function $f$ defined on $M^{n}$ by

$$
\square f=\sum_{i, j}\left[n H\left\langle e_{i}, e_{j}\right\rangle+\left\langle A e_{i}, e_{j}\right\rangle\right]\left(\nabla^{2} f\right)\left(e_{i}, e_{j}\right)=\sum_{i}\left(n H-\lambda_{i}\right)\left(\nabla^{2} f\right)\left(e_{i}, e_{i}\right) .
$$

Note that the following holds at umbilical points:

$$
\square f=\sum_{i}(n-1) H\left(\nabla^{2} f\right)\left(e_{i}, e_{i}\right)=(n-1) H \triangle f .
$$

By the Codazzi equation and [6, Proposition 1], we can prove that the operator $\square$ is selfadjoint relative to the $L^{2}$-inner product of $M^{n}$, that is,

$$
\int_{M^{n}} f(\square g)=\int_{M^{n}}(\square f) g
$$

Naturally, we may ask the following question.

Question 4.1. Can we find other selfadjoint second-order differential operators in terms of the shape operator $\mathscr{A}$, mean curvature, and higher-order mean curvatures?

Fortunately, we do have such a selfadjoint second-order differential operator $\mathscr{L}_{k}$ for each $k=0,1, \ldots, n-1$. The idea is contained in [15, 17]. Following [3], we introduce the $k$ th Newton transformation $T_{k}$ associated to the shape operator $\mathscr{A}$ :

$$
T_{k}=\sum_{i=0}^{k}\left(\begin{array}{l}
n \\
i
\end{array}\right) H_{i} \mathscr{A}^{k-i},
$$


or inductively,

$$
T_{0}=I, \quad T_{k}=\left(\begin{array}{l}
n \\
k
\end{array}\right) H_{k} I+\mathscr{A} T_{k-1} .
$$

It follows from (2.12) that $T_{n}=0$. Since the shape operator $\mathscr{A}$ is selfadjoint, it follows easily that the Newton transformations $T_{k}$ 's are selfadjoint. Clearly, the orthonormal basis $\left\{e_{1}, \ldots, e_{n}\right\}$ diagonalizes the Newton transformations $T_{k}$ 's since it diagonalizes the shape operator $\mathscr{A}$.

Proposition 4.2. If the shape operator $\mathscr{A}$ is negative definite, the Newton transformations $T_{k}$ 's, $k=0,1, \ldots, n-1$, are positive definite.

Proof. Since the shape operator $\mathscr{A}$ is negative definite, all $\lambda_{i}>0$. Without loss of generality, to prove that $T_{k}$ is positive definite, we only need to prove that $\left\langle T_{k} e_{1}, e_{1}\right\rangle>0$. Let $\lambda_{i}^{\prime}=\lambda_{i} / \lambda_{1}, i=1, \ldots, n$, then we have

$$
\begin{aligned}
\left\langle T_{k} e_{1}, e_{1}\right\rangle & =\sum_{i=0}^{k}\left(\begin{array}{l}
n \\
i
\end{array}\right) H_{i}\left(-\lambda_{1}\right)^{k-i} \\
& =\sum_{i=0}^{k} \sigma_{i}\left(\lambda_{1}, \ldots, \lambda_{n}\right)\left(-\lambda_{1}\right)^{k-i} \\
& =\lambda_{1}^{k} \sum_{i=0}^{k}(-1)^{k-i} \sigma_{i}\left(1, \lambda_{2}^{\prime}, \ldots, \lambda_{n}^{\prime}\right) .
\end{aligned}
$$

Now we prove that

$$
\sum_{i=0}^{k}(-1)^{k-i} \sigma_{i}\left(1, x_{2}, \ldots, x_{n}\right)>0, \quad k=0,1, \ldots, n-1,
$$

by induction on $n$, where $x_{2}, \ldots, x_{n}>0$. Clearly, (4.10) holds for $k=0$ or $n=1$. Now assume that $m>1,0<l \leq m-1$, and (4.10) holds for all $n<m$ and all $k<l$ for $n=m$. Let $n=m$ and $k=l$, then we have

$$
\begin{aligned}
\sum_{i=0}^{k}(-1)^{k-i} \sigma_{i}\left(1, x_{2}, \ldots, x_{n}\right)= & \sum_{i=0}^{k}(-1)^{k-i} \sigma_{i}\left(1, x_{2}, \ldots, x_{n-1}\right) \\
& +x_{n} \sum_{i=1}^{k}(-1)^{k-i} \sigma_{i-1}\left(1, x_{2}, \ldots, x_{n}\right) \\
= & \sum_{i=0}^{k}(-1)^{k-i} \sigma_{i}\left(1, x_{2}, \ldots, x_{n-1}\right) \\
& +x_{n} \sum_{i=0}^{k-1}(-1)^{k-1-i} \sigma_{i}\left(1, x_{2}, \ldots, x_{n}\right)>0
\end{aligned}
$$

by the inductive assumption and the fact that $\sum_{i=0}^{k}(-1)^{k-i} \sigma_{i}\left(1, x_{2}, \ldots, x_{n-1}\right)=0$ for $k=$ $n-1$. This completes the proof. 
2062 Compact space-like hypersurfaces in de Sitter space

The following algebraic properties of $T_{k}$ can be easily established from the definitions.

$$
\begin{aligned}
\operatorname{tr} T_{k} & =(n-k)\left(\begin{array}{l}
n \\
k
\end{array}\right) H_{k}=n\left(\begin{array}{c}
n-1 \\
k
\end{array}\right) H_{k} \\
\operatorname{tr}\left(T_{k} \mathscr{A}\right) & =-(k+1)\left(\begin{array}{c}
n \\
k+1
\end{array}\right) H_{k+1}=-n\left(\begin{array}{c}
n-1 \\
k
\end{array}\right) H_{k+1}, \\
\operatorname{tr}\left(T_{k} \mathscr{A}^{2}\right) & =n\left(\begin{array}{c}
n \\
k+1
\end{array}\right) H H_{k+1}-(k+2)\left(\begin{array}{c}
n \\
k+2
\end{array}\right) H_{k+2} \\
& =n\left(\begin{array}{c}
n \\
k+1
\end{array}\right) H H_{k+1}-n\left(\begin{array}{c}
n-1 \\
k+1
\end{array}\right) H_{k+2} .
\end{aligned}
$$

One can also easily derive the identities

$$
\operatorname{tr}\left(T_{k} \nabla_{v} \mathscr{A}\right)=-\left(\begin{array}{c}
n \\
k+1
\end{array}\right)\left\langle\nabla H_{k+1}, v\right\rangle
$$

where $v$ is any vector field tangent to $M^{n}$. Now for each $k=0,1, \ldots, n-1$, we can define a second-order differential operator $\mathscr{L}_{k}$ acting on any $C^{2}$-function $f$ defined on $M^{n}$ by

$$
\mathscr{L}_{k} f=\operatorname{div}\left(T_{k} \nabla f\right) .
$$

It can be easily seen that the operators $\mathscr{L}_{k}$ 's are selfadjoint. Clearly when $k=0$, the operator $\mathscr{L}_{0}$ is the Laplace operator $\Delta=\operatorname{div} \circ \nabla$. Later, we will see that when $k=1$, the operator $\mathscr{L}_{1}$ is the Cheng-Yau operator $\square$.

Finally, we can easily derive the following useful expression for $\mathscr{L}_{k}$ (see [3]):

$$
\mathscr{L}_{k} f=\sum_{i}\left\langle T_{k} \nabla_{e_{i}} \nabla f, e_{i}\right\rangle=\sum_{i}\left\langle T_{k} e_{i}, e_{i}\right\rangle \nabla^{2} f\left(e_{i}, e_{i}\right)
$$

for any $C^{2}$-function $f$ defined on $M^{n}$.

Remark 4.3. More specifically,

$$
\mathscr{L}_{k} f=\sum_{i} \sum_{j=0}^{k}\left(\begin{array}{l}
n \\
j
\end{array}\right) H_{j}\left(-\lambda_{i}\right)^{k-j} \nabla^{2} f\left(e_{i}, e_{i}\right) .
$$

Clearly when $k=1$, the operator $\mathscr{L}_{1}$ is the Cheng-Yau operator $\square=\sum_{i}\left(n H-\lambda_{i}\right) \nabla^{2}$. Note that the following holds at umbilical points:

$$
\mathscr{L}_{k} f=\sum_{i} \sum_{j=0}^{k}\left(\begin{array}{l}
n \\
j
\end{array}\right) H_{j}\left(-\lambda_{i}\right)^{k-j} \nabla^{2} f\left(e_{i}, e_{i}\right)=\sum_{i=0}^{k}(-1)^{k-i}\left(\begin{array}{l}
n \\
i
\end{array}\right) \cdot H_{k} \triangle f .
$$


Remark 4.4. When $T_{k}$ is positive definite, the operator $\mathscr{L}_{k}$ is elliptic. In particular, when the shape operator $\mathscr{A}$ is negative definite, the operator $\mathscr{L}_{k}$ is elliptic by proposition 4.2.

\section{Main results}

Let $\phi: M^{n} \rightarrow S_{1}^{n+1}(c), n \geq 2$, be a compact space-like hypersurface in de Sitter space, $N$ a time-like unit normal vector field for $\phi$, and $a \in \mathbb{R}_{1}^{n+2}$ arbitrary. We consider the height function $\langle\phi, a\rangle$ and the function $\langle N, a\rangle$ on $M^{n}$. Using (2.4), (2.7), we can get the following expressions for the gradient and Hessian of the above two functions:

$$
\begin{aligned}
\langle\nabla\langle\phi, a\rangle, v\rangle & =\langle v, a\rangle, \quad\langle\nabla\langle N, a\rangle, v\rangle=-\langle\mathscr{A} v, a\rangle \\
\left(\nabla^{2}\langle\phi, a\rangle\right)(v, w) & =w v\langle\phi, a\rangle-\left(\nabla_{w} v\right)\langle\phi, a\rangle \\
& =-c\langle v, w\rangle\langle\phi, a\rangle-\langle\mathscr{A} v, w\rangle\langle N, a\rangle, \\
\left(\nabla^{2}\langle N, a\rangle\right)(v, w) & =w v\langle N, a\rangle-\left(\nabla_{w} v\right)\langle N, a\rangle \\
& =c\langle\mathscr{A} v, w\rangle\langle\phi, a\rangle+\langle\mathscr{A} v, \mathscr{A} w\rangle\langle N, a\rangle-\left\langle\left(\nabla_{w} \mathscr{A}\right) v, a\right\rangle,
\end{aligned}
$$

where $v, w$ are vector fields tangent to $M^{n}$. Thus, we have

$$
\begin{aligned}
\mathscr{L}_{k}\langle\phi, a\rangle= & \sum_{i} \sum_{j=0}^{k}\left(\begin{array}{l}
n \\
j
\end{array}\right) H_{j}\left(-\lambda_{i}\right)^{k-j} \nabla^{2}\langle\phi, a\rangle\left(e_{i}, e_{i}\right) \\
= & \sum_{i} \sum_{j=0}^{k}\left(\begin{array}{l}
n \\
j
\end{array}\right) H_{j}\left(-\lambda_{i}\right)^{k-j}\left[-c\langle\phi, a\rangle+\lambda_{i}\langle N, a\rangle\right] \\
= & -c \sum_{j=0}^{k}(-1)^{k-j}\left(\begin{array}{c}
n \\
j
\end{array}\right) H_{j} \sum_{i} \lambda_{i}^{k-j} \cdot\langle\phi, a\rangle \\
& +\sum_{j=0}^{k}(-1)^{k-j}\left(\begin{array}{c}
n \\
j
\end{array}\right) H_{j} \sum_{i} \lambda_{i}^{k+1-j} \cdot\langle N, a\rangle \\
= & -c(n-k)\left(\begin{array}{c}
n \\
k
\end{array}\right) H_{k}\langle\phi, a\rangle+(k+1)\left(\begin{array}{c}
n \\
k+1
\end{array}\right) H_{k+1}\langle N, a\rangle \\
= & n\left(\begin{array}{c}
n-1 \\
k
\end{array}\right)\left[\begin{array}{c}
\left.-c H_{k}\langle\phi, a\rangle+H_{k+1}\langle N, a\rangle\right] .
\end{array}\right.
\end{aligned}
$$

Note that the Minkowski formulas in Proposition 2.2 are regained by the selfadjointness of the operators $\mathscr{L}_{k}$ 's. 
2064 Compact space-like hypersurfaces in de Sitter space

For any vector field $v$ tangent to $M^{n}$, we have

$$
\nabla_{v} \nabla\langle N, a\rangle=c \mathscr{A} v\langle\phi, a\rangle+\mathscr{A}^{2} v\langle N, a\rangle-\left(\nabla_{v} \mathscr{A}\right) a^{T},
$$

by the selfadjointness of the operator $\nabla_{v} \mathscr{A}$, where $a^{T}$ is the tangent component of $a$ to $M^{n}$. Thus by (2.8), (4.13), (4.14), and (4.15), we have

$$
\begin{aligned}
\mathscr{L}_{k}\langle N, a\rangle= & \sum_{i}\left\langle T_{k} \nabla_{e_{i}} \nabla\langle N, a\rangle, e_{i}\right\rangle \\
= & \sum_{i}\left\langle c T_{k} \mathscr{A} e_{i}\langle\phi, a\rangle+T_{k} \mathscr{A}^{2} e_{i}\langle N, a\rangle-T_{k}\left(\nabla_{e_{i}} \mathscr{A}\right) a^{T}, e_{i}\right\rangle \\
= & c \operatorname{tr}\left(T_{k} \mathscr{A}\right)\langle\phi, a\rangle+\operatorname{tr}\left(T_{k} \mathscr{A}^{2}\right)\langle N, a\rangle-\sum_{i}\left\langle T_{k}\left(\nabla_{e_{i}} \mathscr{A}\right) a^{T}, e_{i}\right\rangle \\
= & c \operatorname{tr}\left(T_{k} \mathscr{A}\right)\langle\phi, a\rangle+\operatorname{tr}\left(T_{k} \mathscr{A}^{2}\right)\langle N, a\rangle-\sum_{i}\left\langle T_{k}\left(\nabla_{a^{T}} \mathscr{A}\right) e_{i}, e_{i}\right\rangle \\
= & c \operatorname{tr}\left(T_{k} \mathscr{A}\right)\langle\phi, a\rangle+\operatorname{tr}\left(T_{k} \mathscr{A}^{2}\right)\langle N, a\rangle-\operatorname{tr}\left(T_{k} \nabla_{a^{T}} \mathscr{A}\right) \\
= & -c n\left(\begin{array}{c}
n-1 \\
k
\end{array}\right) H_{k+1}\langle\phi, a\rangle+n\left[\left(\begin{array}{c}
n \\
k+1
\end{array}\right) H H_{k+1}-\left(\begin{array}{l}
n-1 \\
k+1
\end{array}\right) H_{k+2}\right]\langle N, a\rangle \\
& +\left(\begin{array}{c}
n \\
k+1
\end{array}\right)\left\langle\nabla H_{k+1}, a^{T}\right\rangle \\
= & -c n\left(\begin{array}{c}
n-1 \\
k
\end{array}\right) H_{k+1}\langle\phi, a\rangle+n\left[\left(\begin{array}{c}
n \\
k+1
\end{array}\right) H H_{k+1}-\left(\begin{array}{c}
n-1 \\
k+1
\end{array}\right) H_{k+2}\right]\langle N, a\rangle \\
& +\left(\begin{array}{c}
n \\
k+1
\end{array}\right)\left\langle\nabla H_{k+1}, a\right\rangle .
\end{aligned}
$$

Remark 5.1. In particular, when $k=0$, we have

$$
\triangle\langle N, a\rangle=\mathscr{L}_{0}\langle N, a\rangle=-c n H\langle\phi, a\rangle+\left[n^{2} H^{2}-n(n-1) H_{2}\right]\langle N, a\rangle+n\langle\nabla H, a\rangle .
$$

Proposition 5.2.

$$
\mathscr{L}_{k}\langle N, a\rangle=-\mathscr{L}_{k+1}\langle\phi, a\rangle+\left(\begin{array}{c}
n \\
k+1
\end{array}\right)\left[H_{k+1} \Delta\langle\phi, a\rangle+\left\langle\nabla H_{k+1}, a\right\rangle\right]
$$

for $k=0,1, \ldots, n-1$.

Proof. By (5.2), we have

$$
\begin{aligned}
\frac{1}{n} H_{k+1} & \triangle\langle\phi, a\rangle-\frac{1}{n\left(\begin{array}{l}
n-1 \\
k+1
\end{array}\right)} \mathscr{L}_{k+1}\langle\phi, a\rangle \\
& =\left(H_{1} H_{k+1}-H_{k+2}\right)\langle N, a\rangle .
\end{aligned}
$$


Thus by (5.2) and (5.4), we have

$$
\begin{aligned}
\mathscr{L}_{k}\langle N, a\rangle= & \frac{\left(\begin{array}{c}
n-1 \\
k
\end{array}\right)}{\left(\begin{array}{c}
n-1 \\
k+1
\end{array}\right)} \mathscr{L}_{k+1}\langle\phi, a\rangle+\left(\begin{array}{c}
n \\
k+1
\end{array}\right)\left[H_{k+1} \Delta\langle\phi, a\rangle-\frac{1}{\left(\begin{array}{c}
n-1 \\
k+1
\end{array}\right)} \mathscr{L}_{k+1}\langle\phi, a\rangle\right] \\
& +\left(\begin{array}{c}
n \\
k+1
\end{array}\right)\left\langle\nabla H_{k+1}, a\right\rangle \\
= & -\mathscr{L}_{k+1}\langle\phi, a\rangle+\left(\begin{array}{c}
n \\
k+1
\end{array}\right)\left[H_{k+1} \triangle\langle\phi, a\rangle+\left\langle\nabla H_{k+1}, a\right\rangle\right] .
\end{aligned}
$$

Theorem 5.3. Let $\phi: M^{n} \rightarrow S_{1}^{n+1}(c), n \geq 2$, be a compact space-like hypersurface in de Sitter space and $0 \leq i<j \leq n-1$, then

$$
\int_{M^{n}}\left[\frac{1}{n\left(\begin{array}{c}
n-1 \\
j
\end{array}\right)} \mathscr{L}_{j} H_{i}-\frac{1}{n\left(\begin{array}{c}
n-1 \\
i
\end{array}\right)} \mathscr{L}_{i} H_{j}\right]\langle\phi, a\rangle+\left(H_{i+1} H_{j}-H_{i} H_{j+1}\right)\langle N, a\rangle=0,
$$

or equivalently,

$$
\int_{M^{n}} \frac{1}{n}\left\langle\frac{1}{\left(\begin{array}{c}
n-1 \\
i
\end{array}\right)} T_{i} \nabla H_{j}-\frac{1}{\left(\begin{array}{c}
n-1 \\
j
\end{array}\right)} T_{j} \nabla H_{i}, a\right\rangle+\left(H_{i+1} H_{j}-H_{i} H_{j+1}\right)\langle N, a\rangle=0,
$$

for any vector $a \in \mathbb{R}_{1}^{n+2}$.

Proof. By (5.2), we have

$$
\frac{1}{n\left(\begin{array}{c}
n-1 \\
i
\end{array}\right)} H_{j} \mathscr{L}_{i}\langle\phi, a\rangle-\frac{1}{n\left(\begin{array}{c}
n-1 \\
j
\end{array}\right)} H_{i} \mathscr{L}_{j}\langle\phi, a\rangle=\left(H_{i+1} H_{j}-H_{i} H_{j+1}\right)\langle N, a\rangle .
$$

Thus,

$$
\int_{M^{n}} \frac{1}{n\left(\begin{array}{c}
n-1 \\
i
\end{array}\right)} H_{j} \mathscr{L}_{i}\langle\phi, a\rangle-\frac{1}{n\left(\begin{array}{c}
n-1 \\
j
\end{array}\right)} H_{i} \mathscr{L}_{j}\langle\phi, a\rangle=\int_{M^{n}}\left(H_{i+1} H_{j}-H_{i} H_{j+1}\right)\langle N, a\rangle .
$$

Since the operators $\mathscr{L}_{k}$ 's are selfadjoint, we have

$$
\int_{M^{n}}\left[\frac{1}{n\left(\begin{array}{c}
n-1 \\
j
\end{array}\right)} \mathscr{L}_{j} H_{i}-\frac{1}{n\left(\begin{array}{c}
n-1 \\
i
\end{array}\right)} \mathscr{L}_{i} H_{j}\right]\langle\phi, a\rangle+\left(H_{i+1} H_{j}-H_{i} H_{j+1}\right)\langle N, a\rangle=0,
$$

or equivalently,

$$
\int_{M^{n}} \frac{1}{n}\left\langle\frac{1}{\left(\begin{array}{c}
n-1 \\
i
\end{array}\right)} T_{i} \nabla H_{j}-\frac{1}{\left(\begin{array}{c}
n-1 \\
j
\end{array}\right)} T_{j} \nabla H_{i}, a\right\rangle+\left(H_{i+1} H_{j}-H_{i} H_{j+1}\right)\langle N, a\rangle=0
$$

since the operators $\mathscr{L}_{k}=\operatorname{div} \circ T_{k} \nabla$, for any vector $a \in \mathbb{R}_{1}^{n+2}$. 
Theorem 5.4. Let $\phi: M^{n} \rightarrow S_{1}^{n+1}(c), n \geq 2$, be a compact space-like hypersurface in de Sitter space, $a \in \mathbb{R}_{1}^{n+2}$ any unit time-like vector with the same time-orientation as $N$, and $0 \leq k \leq n-2$, then

$$
\int_{M^{n}}\left\langle\left(\begin{array}{c}
n-1 \\
k+1
\end{array}\right) T_{k} \nabla H_{k+1}-\left(\begin{array}{c}
n-1 \\
k
\end{array}\right) T_{k+1} \nabla H_{k}, a\right\rangle \geq 0,
$$

and the equality holds if and only if $M^{n}$ is totally umbilical when $k=0$, or additionally if $H_{k+1}^{2}+H_{k}^{2} H_{k+2}^{2} \neq 0$ when $1 \leq k \leq n-2$.

Proof. For any unit time-like vector $a \in \mathbb{R}_{1}^{n+2}$ with the same time orientation as $N$, that is, $|x|^{2}=-1$ and $x_{0} \geq 1$, we have $\langle N, a\rangle \leq-1$. Thus by taking $i=k, j=k+1$ in Theorem 5.3 and Proposition 3.2, we can deduce that

$$
\int_{M^{n}}\left\langle\left(\begin{array}{c}
n-1 \\
k+1
\end{array}\right) T_{k} \nabla H_{k+1}-\left(\begin{array}{c}
n-1 \\
k
\end{array}\right) T_{k+1} \nabla H_{k}, a\right\rangle \geq 0,
$$

and the equality holds if and only if $M^{n}$ is totally umbilical when $k=0$ or additionally if $H_{k+1}^{2}+H_{k}^{2} H_{k+2}^{2} \neq 0$ when $1 \leq k \leq n-2$.

Remark 5.5. In particular, when $k=0$, we have

$$
\int_{M^{n}}\langle\nabla H, a\rangle \geq 0
$$

and the equality holds if and only if $M^{n}$ is totally umbilical for any unit time-like vector $a \in \mathbb{R}_{1}^{n+2}$ with the same time orientation as $N$.

Remark 5.6. In particular, if $H_{k}$ and $H_{k+1}$ are constant, $0 \leq k \leq n-2$, then $M^{n}$ is totally umbilical when $k=0$, or additionally if $H_{k+1}^{2}+H_{k}^{2} H_{k+2}^{2} \neq 0$ when $1 \leq k \leq n-2$. See also [3].

Theorem 5.7. Let $\phi: M^{n} \rightarrow S_{1}^{n+1}(c), n \geq 2$, be a compact space-like hypersurface in de Sitter space with $H_{1} \geq 0, \ldots, H_{n} \geq 0, a \in \mathbb{R}_{1}^{n+2}$ any unit time-like vector with the same time orientation as $N$, and $0 \leq i<j \leq n-1, j \geq i+2$, then

$$
\int_{M^{n}}\left\langle\left(\begin{array}{c}
n-1 \\
j
\end{array}\right) T_{i} \nabla H_{j}-\left(\begin{array}{c}
n-1 \\
i
\end{array}\right) T_{j} \nabla H_{i}, a\right\rangle \geq 0 .
$$

Moreover, if $\sum_{k=n-j+1}^{n} H_{k}^{2} \neq 0$, then the equality holds if and only if $M^{n}$ is totally umbilical.

Proof. For any unit time-like vector $a \in \mathbb{R}_{1}^{n+2}$ with the same time orientation as $N$, we have $\langle N, a\rangle \leq-1$. Thus by Theorems 5.3 and 3.7 , we can deduce that

$$
\int_{M^{n}}\left\langle\left(\begin{array}{c}
n-1 \\
j
\end{array}\right) T_{i} \nabla H_{j}-\left(\begin{array}{c}
n-1 \\
i
\end{array}\right) T_{j} \nabla H_{i}, a\right\rangle \geq 0,
$$

and when $\sum_{k=n-j+1}^{n} H_{k}^{2} \neq 0$, the equality holds if and only if $M^{n}$ is totally umbilical. 
Corollary 5.8. Let $\phi: M^{n} \rightarrow S_{1}^{n+1}(c), n \geq 2$, be a compact space-like hypersurface in de Sitter space with $H_{1} \geq 0, \ldots, H_{n} \geq 0$ and constant $\sum_{i=1}^{k-1} a_{i} H_{i}+H_{k}, a_{i} \geq 0,2 \leq k \leq n-1$. If $\sum_{i=n-k+1}^{n} H_{i}^{2} \neq 0$, then $M^{n}$ is totally umbilical.

Proof. Fix a unit time-like vector $a \in \mathbb{R}_{1}^{n+2}$ with the same time orientation as $N$. By Theorems 5.4 and 5.7, we have

$$
\int_{M^{n}}\left\langle\nabla H_{i}, a\right\rangle \geq 0, \quad i=1, \ldots, k .
$$

Since

$$
0=\int_{M^{n}}\left\langle\nabla\left(\sum_{i=1}^{k-1} a_{i} H_{i}+H_{k}\right), a\right\rangle=\sum_{i=1}^{k-1} a_{i} \int_{M^{n}}\left\langle\nabla H_{i}, a\right\rangle+\int_{M^{n}}\left\langle\nabla H_{k}, a\right\rangle \geq 0,
$$

we have

$$
\int_{M^{n}}\left\langle\nabla H_{k}, a\right\rangle=0
$$

Thus, $M^{n}$ is totally umbilical by Theorem 5.7 .

THEOREM 5.9. Let $\phi: M^{n} \rightarrow S_{1}^{n+1}(c), n \geq 2$, be a compact space-like hypersurface in de Sitter space with $H_{k+1}>0, a \in \mathbb{R}_{1}^{n+2}$ any unit time-like vector with the same time orientation as $N$, and $0 \leq i<j \leq k \leq n-1, j \geq i+2$. If there exists a point of $M^{n}$, where $H_{1}, \ldots, H_{k}$ are positive, then

$$
\int_{M^{n}}\left\langle\left(\begin{array}{c}
n-1 \\
j
\end{array}\right) T_{i} \nabla H_{j}-\left(\begin{array}{c}
n-1 \\
i
\end{array}\right) T_{j} \nabla H_{i}, a\right\rangle \geq 0
$$

with equality if and only if $M^{n}$ is totally umbilical.

Proof. For any unit time-like vector $a \in \mathbb{R}_{1}^{n+2}$ with the same time orientation as $N$, we have $\langle N, a\rangle \leq-1$. Thus by Theorems $5.3,3.6$, and 3.8 , we can deduce that

$$
\int_{M^{n}}\left\langle\left(\begin{array}{c}
n-1 \\
j
\end{array}\right) T_{i} \nabla H_{j}-\left(\begin{array}{c}
n-1 \\
i
\end{array}\right) T_{j} \nabla H_{i}, a\right\rangle \geq 0,
$$

and the equality holds if and only if $M^{n}$ is totally umbilical.

Let $a \in \mathbb{R}_{1}^{n+2}$ be a unit time-like vector. The intersection of $S_{1}^{n+1}(c) \subset \mathbb{R}_{1}^{n+2}$ and the space-like hyperplane $\left\{x \in \mathbb{R}_{1}^{n+2} \mid\langle x, a\rangle=0\right\}$ defines an $n$-sphere which is a totally geodesic hypersurface in $S_{1}^{n+1}(c)$. We will refer to that sphere as the equator of $S_{1}^{n+1}(c)$ determined by $a$. This equator divides the de Sitter space into two connected components; the future which is given by

$$
\left\{x \in \mathbb{R}_{1}^{n+2} \mid\langle x, a\rangle<0\right\},
$$


and the past given by

$$
\left\{x \in \mathbb{R}_{1}^{n+2} \mid\langle x, a\rangle>0\right\}
$$

Following [3], we can easily get the following corollary.

Corollary 5.10. Let $\phi: M^{n} \rightarrow S_{1}^{n+1}(c), n \geq 2$, be a compact space-like hypersurface in de Sitter space and $2 \leq k \leq n-1$. If $M^{n}$ is contained in the chronological future (or past) relative to the equator of $S_{1}^{n+1}(c)$ determined by a unit time-like vector $a \in \mathbb{R}_{1}^{n+2}$ with the same time orientation as $N$ and $H_{k+1}>0\left(\right.$ or $\left.(-1)^{k+1} H_{k+1}>0\right)$, then

$$
\int_{M^{n}}\left\langle\nabla H_{i}, a\right\rangle \geq 0 \quad\left(\text { or }(-1)^{i+1} \int_{M^{n}}\left\langle\nabla H_{i}, a\right\rangle \geq 0\right), \quad 2 \leq i \leq k,
$$

with each equality if and only if $M^{n}$ is totally umbilical.

Proof. First we prove the future case. By Theorem 5.9, it is sufficient to prove that there exists a point of $M^{n}$, where all $H_{i}>0$. Since $M^{n}$ is contained in the chronological future relative to the equator determined by $a$ and $M^{n}$ is compact, there exists a point $x_{0} \in M^{n}$ such that

$$
\max _{x \in M^{n}}\langle\phi(x), a\rangle=\left\langle\phi\left(x_{0}\right), a\right\rangle<0 .
$$

Thus by maximum principle, we have

$$
\begin{aligned}
-c\left\langle\phi\left(x_{0}\right), a\right\rangle+\lambda_{i}\left\langle N\left(x_{0}\right), a\right\rangle & =-c\left\langle e_{i}, e_{i}\right\rangle\left\langle\phi\left(x_{0}\right), a\right\rangle-\left\langle A e_{i}, e_{i}\right\rangle\left\langle N\left(x_{0}\right), a\right\rangle \\
& =\nabla^{2}\langle\phi, a\rangle\left(e_{i}, e_{i}\right) \leq 0 .
\end{aligned}
$$

Since $a \in \mathbb{R}_{1}^{n+2}$ is a unit time-like vector with the same time orientation as $N$, we have $\langle N, a\rangle \leq-1$. So

$$
\lambda_{i} \geq c \frac{\left\langle\phi\left(x_{0}\right), a\right\rangle}{\left\langle N\left(x_{0}\right), a\right\rangle}>0, \quad i=1, \ldots, n
$$

Thus all $H_{i}>0$. For the past case, we only need to replace $N$ and $a$ by $-N$ and $-a$, respectively, and the proof for the future case applies. This completes the proof.

\section{References}

[1] K. Akutagawa, On spacelike hypersurfaces with constant mean curvature in the de Sitter space, Math. Z. 196 (1987), no. 1, 13-19.

[2] J. A. Aledo and L. J. Alías, On the volume and the Gauss map image of spacelike hypersurfaces in de Sitter space, Proc. Amer. Math. Soc. 130 (2002), no. 4, 1145-1151.

[3] J. A. Aledo, L. J. Alías, and A. Romero, Integral formulas for compact space-like hypersurfaces in de Sitter space: applications to the case of constant higher order mean curvature, J. Geom. Phys. 31 (1999), no. 2-3, 195-208.

[4] H.-D. Cao, Y. Shen, and S. Zhu, A Bernstein theorem for complete spacelike constant mean curvature hypersurfaces in Minkowski space, Calc. Var. Partial Differential Equations 7 (1998), no. 2, 141-157. 
[5] Q.-M. Cheng and S. Ishikawa, Spacelike hypersurfaces with constant scalar curvature, Manuscripta Math. 95 (1998), no. 4, 499-505.

[6] S. Y. Cheng and S. T. Yau, Hypersurfaces with constant scalar curvature, Math. Ann. 225 (1977), no. 3, 195-204.

[7] A. J. Goddard, Some remarks on the existence of spacelike hypersurfaces of constant mean curvature, Math. Proc. Cambridge Philos. Soc. 82 (1977), no. 3, 489-495.

[8] G. H. Hardy, J. E. Littlewood, and G. Pólya, Inequalities, 2nd ed., Cambridge Mathematical Library, Cambridge University Press, Cambridge, 1988.

[9] S.-E. Koh, A characterization of round spheres, Proc. Amer. Math. Soc. 126 (1998), no. 12, 36573660.

[10] Sphere theorem by means of the ratio of mean curvature functions, Glasgow Math. J. 42 (2000), no. 1, 91-95.

[11] S. Montiel, An integral inequality for compact spacelike hypersurfaces in de Sitter space and applications to the case of constant mean curvature, Indiana Univ. Math. J. 37 (1988), no. 4, 909-917.

[12] S. Montiel and A. Ros, Compact hypersurfaces: the Alexandrov theorem for higher order mean curvatures, Differential Geometry (B. Lawson, ed.), Pitman Monogr. Surveys Pure Appl. Math., vol. 52, Longman Scientific and Technical, Harlow, 1991, pp. 279-296.

[13] B. O'Neill, Semi-Riemannian Geometry, Pure and Applied Mathematics, vol. 103, Academic Press, New York, 1983.

[14] J. Ramanathan, Complete spacelike hypersurfaces of constant mean curvature in de Sitter space, Indiana Univ. Math. J. 36 (1987), no. 2, 349-359.

[15] R. C. Reilly, Variational properties of functions of the mean curvatures for hypersurfaces in space forms, J. Differential Geometry 8 (1973), 465-477.

[16] A. Ros, Compact hypersurfaces with constant higher order mean curvatures, Rev. Mat. Iberoamericana 3 (1987), no. 3-4, 447-453.

[17] H. Rosenberg, Hypersurfaces of constant curvature in space forms, Bull. Sci. Math. 117 (1993), no. 2, 211-239.

Jinchi Lv: Department of Mathematics, Princeton University, Princeton, NJ 08544-1000, USA

E-mail address: jlv@math.princeton.edu 


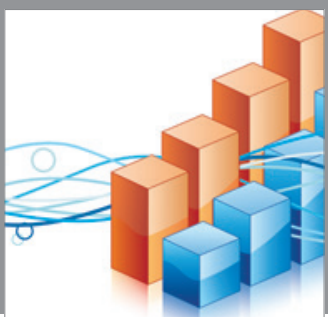

Advances in

Operations Research

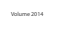

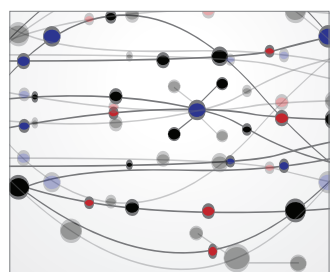

\section{The Scientific} World Journal
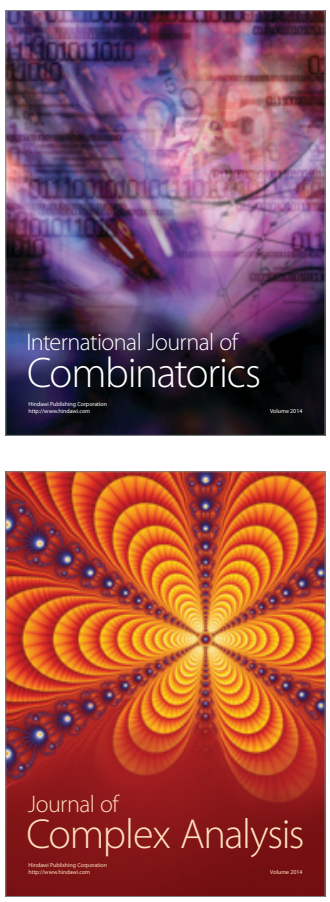

International Journal of

Mathematics and

Mathematical

Sciences
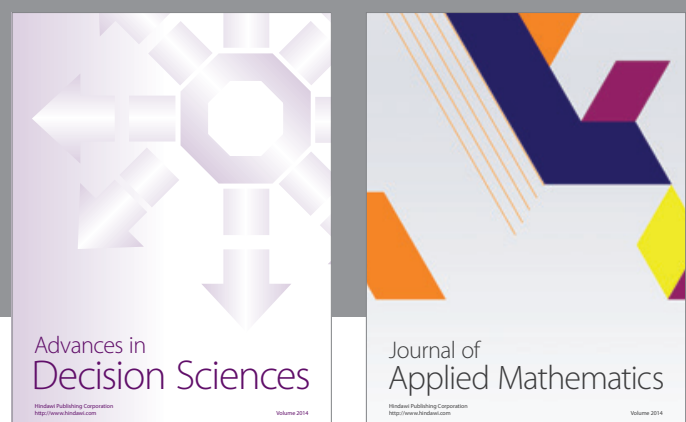

Journal of

Applied Mathematics
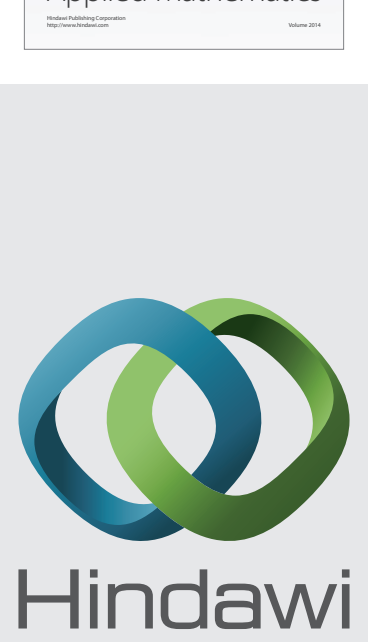

Submit your manuscripts at http://www.hindawi.com
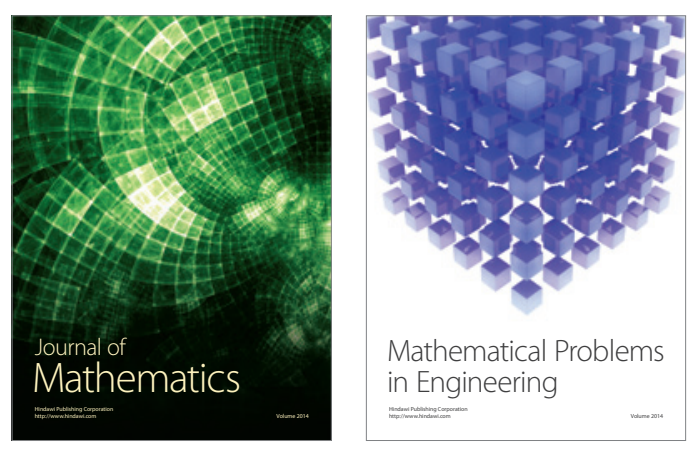

Mathematical Problems in Engineering
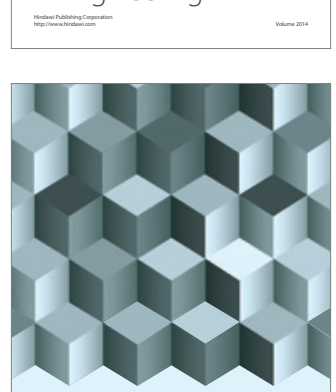

Journal of

Function Spaces
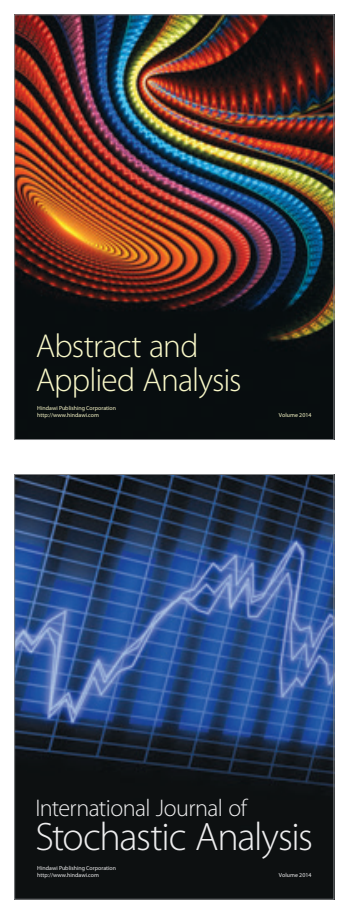

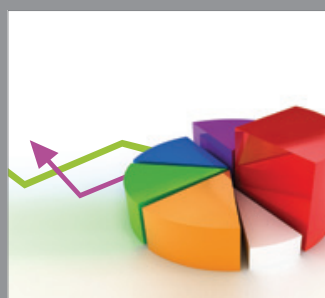

ournal of

Probability and Statistics

Promensencen
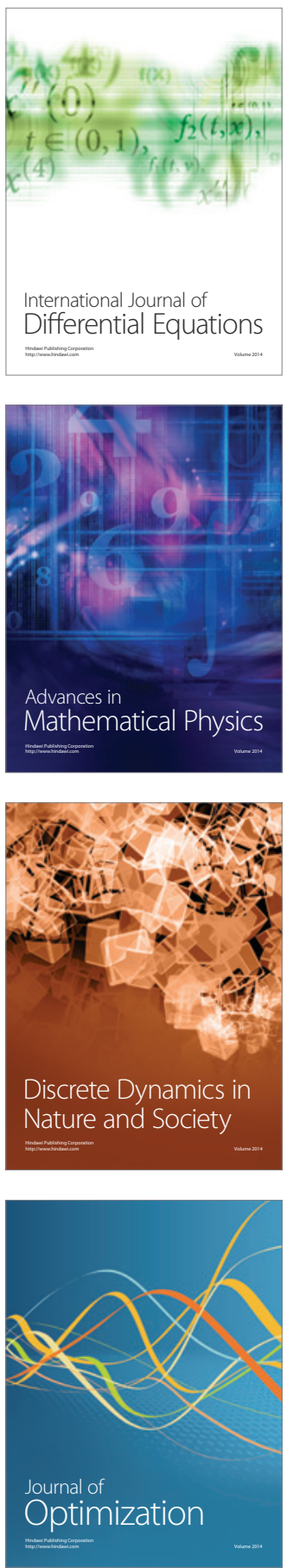